\title{
Noninvasive Photochemical Sealing for Achilles Tendon Rupture by Combination of Upconversion Nanoparticles and Photochemical Tissue Bonding Technology
}

\author{
Yiming Zhu, ${ }^{1}$ Aiguo Xie $\mathbb{D}^{2},{ }^{2}$ Ming $\mathrm{Li}^{2}$ Chihao Zhang $\mathbb{D}^{1},{ }^{1}$ and Tao Ni $\mathbb{D}^{2}$ \\ ${ }^{1}$ Department of General Surgery, Shanghai Ninth People's Hospital, School of Medicine, Shanghai Jiao Tong University, \\ Shanghai 200011, China \\ ${ }^{2}$ Department of Plastic and Reconstructive Surgery, Shanghai Ninth People's Hospital, School of Medicine, Shanghai Jiao \\ Tong University, Shanghai 200011, China
}

Correspondence should be addressed to Tao Ni; prsnt123456@126.com

Received 10 December 2019; Revised 31 March 2020; Accepted 15 April 2020; Published 19 May 2020

Academic Editor: Ali I. Abdalla

Copyright (c) 2020 Yiming Zhu et al. This is an open access article distributed under the Creative Commons Attribution License, which permits unrestricted use, distribution, and reproduction in any medium, provided the original work is properly cited.

Photochemical tissue bonding (PTB), based on photosensitizer rose bengal (RB) and green light, has been regarded as an effective alternative to surgical suture and has been reported to provide benefits for Achilles tendon repair. Limited to the poor penetration of green light, secondary damage still exists while applying PTB for closed Achilles tendon rupture. This study is aimed at exploring the effects of noninvasive photochemical sealing on Achilles tendon rupture by the combination of PTB and upconversion nanoparticles (UCNPs). The rare-earth UCNPs of $\mathrm{NaYF}_{4}: \mathrm{Yb} / \mathrm{Er}(\mathrm{Y}: \mathrm{Yb}: \mathrm{Er}=78: 20: 2)$ were fabricated and then loaded into Chitosan/ $\beta$-GP hydrogel containing RB to prepare UCNPs@RB/Chitosan/ $\beta$-GP hydrogel. The properties of UCNPs and UCNP/Chitosan/ $\beta$-GP hydrogel were characterized by TEM, SEM, DLS, and FTIR analysis. The effects of UCNP and PTB combination were evaluated in an Achilles tendon rupture rat model using histological analysis. Bioluminescence imaging of ROS was performed to explore the potential mechanism. UCNPs had a uniform shape with a diameter of $29.7 \pm 2.6 \mathrm{~nm}$. The UCNPs@RB/Chitosan/ $\beta$-GP hydrogel could upconvert the near-infrared light into green light. The results of histological assessment showed that compared with traditional suture repair, the rats injected with UCNPs@RB/Chitosan/ $\beta$-GP hydrogel followed by irradiating with near-infrared light and the rats treated with RB solution followed by irradiating with green light had better effects on Achilles tendon repair. The benefits might be related to the generation of ROS in the PTB process. These findings indicated that the combination of PTB and UCNPs@RB/Chitosan/ $\beta$-GP hydrogel could be used as a noninvasive photochemical sealing for Achilles tendon rupture.

\section{Introduction}

Achilles tendon rupture is widely reported, and its incidence increases every year. In Denmark, the incidence of acute Achilles tendon rupture increased from 26.95 per 10,000 persons per year in 1994 to 31.17 per 10,000 persons per year in 2013 [1]. Operative and nonoperative methods are broadly used for the treatment of Achilles tendon rupture. Compared with the nonoperative treatment, the operative treatment had a lower risk of rerupture [2-5]. However, operative therapy increased the risk of many complications, including infec- tion, adhesions, sural nerve injury, deep vein thrombosis, and disturbed skin sensibility [2-5].

Photochemical tissue bonding (PTB), which was first developed by the researchers from Massachusetts General Hospital, Harvard Medical School, is a novel suture-less technique for tissue repair and is regarded as an effective alternative to surgical suture [6]. The mechanisms of PTB depend on two components: photosensitizer rose bengal (RB) and green light. RB could absorb the photons of green laser and thus generate ROS reacting with amino acids in structural proteins, in which covalent binding between proteins, including 
collagen crosslinking, will be promoted [7]. Chan et al. has reported that PTB promotes tendon repair at early stages in a full thickness Achilles tendon transection rat model [8]. In our previous study, we found that a combination of PTB and electrospun silk provided benefits for tendon healing in an Achilles tendon transection rabbit model [9]. Recently, PTB has been reported to bond human amniotic membrane with the surface of the flexor digitorum profundus tendon and thus promote hand tendon repair better than surgical suture [10]. All these reports suggest that PTB can be a potential surgical technology for ruptured tendon repair. However, limited to the poor penetration of green light while applying PTB technology in closed tendon rupture injury, an incision to the skin to expose the ruptured tendon site is still needed, which will cause secondary damage. Whether there is a noninvasive sealing approach for the repair of ruptured tendon repair attracts our attention.

Upconversion nanoparticles (UCNPs), which can convert radiation with a longer wavelength (e.g., infrared or near-infrared light) to radiation with a shorter wavelength (e.g., visible or ultraviolet light) by a multiphoton mechanism, have attracted considerable attention for their application in medicine [11]. Based on the properties of UCNPs, Han et al. had reported the effective effect of the combination of PTB with UCNPs for the repair of dissected skin tissues [12]. Thus, if we convert the penetrating near-infrared light to green light by UCNPs, secondary damage will be avoided in the application of PTB in tendon repair. As a natural component of crab or shrimp shells, Chitosan is a biologically compatible and degradable polymer [13]. The aqueous formulations of Chitosan containing $\beta$-glycerophosphate (Chitosan/ $\beta$-GP) can be easily administered by injection, and when injected in vivo, Chitosan/ $\beta$-GP undergoes solution-gel transition at a temperature close to $37^{\circ} \mathrm{C}[13$, 14]. The resulting Chitosan/ $\beta$-GP hydrogel possesses superior biocompatibility [14]. Thus, Chitosan/ $\beta$-GP hydrogel was applied to load UCNPs.

Here, we prepared an injectable Chitosan/ $\beta$-GP hydrogel to load RB and UCNPs, which could convert near-infrared light to green light. The resulting solutions were injected into the site of a transected Achilles tendon and then turned into hydrogel in situ. Then, near-infrared light irradiation was used at the transected site through the skin. The effects of UCNP and PTB combination on Achilles tendon repair in rats were evaluated by Tunel assay, hematoxylin-eosin ( $\mathrm{H} \& \mathrm{E})$ staining, and Masson's trichrome staining.

\section{Materials and Methods}

2.1. Preparation of UCNPs and Injectable UCNP/RB/Chitosan Hydrogel. $\mathrm{La}_{2} \mathrm{O}_{3}, \mathrm{Yb}_{2} \mathrm{O}_{3}, \mathrm{Y}_{2} \mathrm{O}_{3}$, and $\mathrm{Er}_{2} \mathrm{O}_{3}$ were completely dissolved in $10 \% \mathrm{HCl}$ under heating conditions to form hexahydrate chloride. After drying in vacuum, the abovementioned hexahydrate chloride was dissolved in ultrapure water and ethanol and then $\mathrm{n}$-hexane was added. A reflux reaction was performed at $80^{\circ} \mathrm{C}$ for $4 \mathrm{~h}$. The mixture was divided into two layers. The lower layer was removed by a separation funnel. The upper layer was washed with water for several times followed by drying in vacuum to obtain a waxy substance. A rare-earth oleic acid precursor was obtained by dissolving the above waxy substance in oleic acid under nitrogen circumstance. Then, equal amounts of the rare-earth oleic acid precursor and sodium oleate were dissolved in oleic acid and stirred violently to form a uniform solution. The uniform solution was mixed with $\mathrm{BmimBF}_{4}$ followed by heating in a hydrothermal reactor for $24 \mathrm{~h}$ at $240^{\circ} \mathrm{C}$. After cooling at room temperature, the UCNPs of $\mathrm{NaYF}_{4}: \mathrm{Yb} / \mathrm{Er}$ were generated at the junction of the oil phase and ionic liquid phase. Finally, the UCNPs were collected and washed alternately with cyclohexane and ethanol for several times. After drying at $240^{\circ} \mathrm{C}$ for $12 \mathrm{~h}$ in vacuum, the prepared UCNPs were stored for subsequent experiments.

For the preparation of UCNPs@RB/Chitosan/ $\beta$-GP hydrogel, Chitosan chloride, beta-glycerin sodium phosphate $(\beta$-GP), hydroxyethyl cellulose (HEC), and UCNPs were dissolved and dispersed in ultrapure water at $4^{\circ} \mathrm{C}(\mathrm{pH}=8)$. Then, after the addition of $\mathrm{RB}$, the aqueous solution of UCNPs@RB/Chitosan/ $\beta$-GP was obtained. The above solutions turned into gel in the mold at $37^{\circ} \mathrm{C}$.

\subsection{Characterization of UCNPs and UCNP/Chitosan/ $\beta-G P$} Hydrogel. The morphological structure of UCNPs was studied by JEM-2100 transmission electron microscopy (TEM; JEOL, Tokyo, Japan). The particle size of UCNPs was detected by dynamic light-scattering assay with a Malvern Zetasizer NanoZS instrument. The morphological structure of UCNP/Chitosan/ $\beta$-GP hydrogel was studied by JSM7001F scanning electron microscopy (SEM; JEOL, Tokyo, Japan). Fourier transform infrared spectroscopy (FTIR) analysis was performed to analyze the intermolecular interactions among various components of UCNP/Chitosan/ $\beta-\mathrm{GP}$ hydrogel with a scan range from 4000 to $650 \mathrm{~cm}^{-1}$ on a Nicolet 5700 spectrophotometer (Thermo Fisher Scientific, Waltham, MA, USA).

2.3. Cell Culture. The rat tenocytes were maintained in DMEM containing $10 \%$ fetal bovine serum and $1 \%$ penicillinstreptomycin and cultured at $37^{\circ} \mathrm{C}$ and $5 \% \mathrm{CO}_{2}$.

2.4. Cytotoxicity Assay. The cytotoxicity of UCNP/RB/Chito$\mathrm{san} / \beta$-GP hydrogel on tenocytes was determined by CCK- 8 assay. Tenocytes were cultured on the surface of UCNP/RB/Chitosan hydrogel. After having been incubated with Chitosan/ $\beta$-GP, RB/Chitosan/ $\beta$-GP, and UCNP/RB/Chitosan/ $\beta$ GP hydrogel for 1,4 , and 7 days, the tenocytes were planted into a 96-well plate.

2.5. Achilles Tendon Injury Rat Model. The experiments were approved by the Research Animal Care Subcommittee of Shanghai Ninth People's Hospital, School of Medicine, Shanghai Jiao Tong University. Male Sprague Dawley rats (220-250 g) were obtained from Shanghai Jiesijie Experimental Animal Co., Ltd. (Shanghai, China). All rats were divided into four groups: the normal group; the suture repair group (SR group); the RB+532 nm group; and the UCNPs@hydrogel+808 $\mathrm{nm}$ group.

The rats were anesthetized by intraperitoneal injection of $2 \%$ pentobarbital sodium $(2.5 \mathrm{~mL} / \mathrm{kg}$ weight $)$. The Achilles tendon in the right hind limb was exposed and partly 


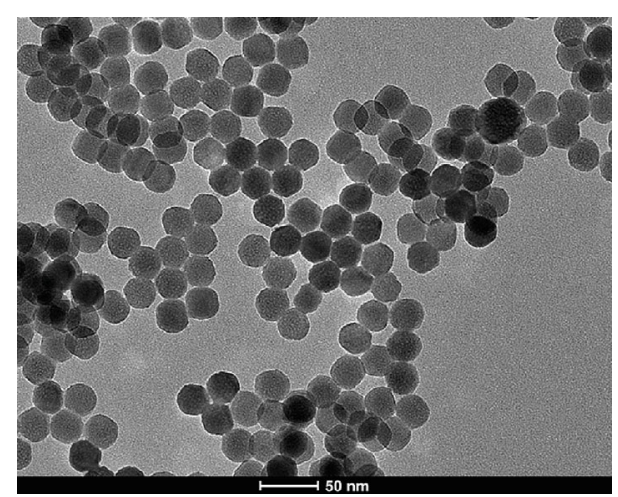

(a)

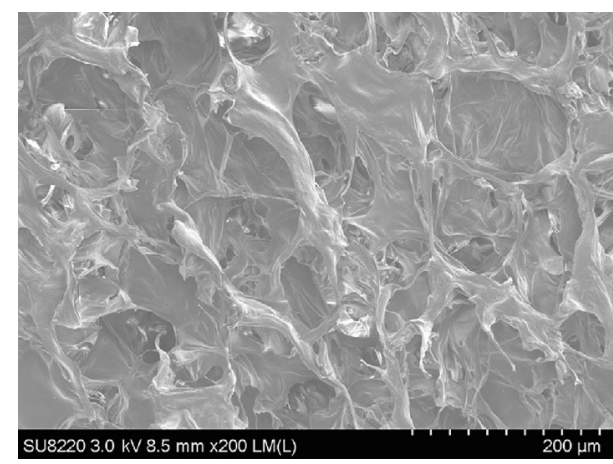

(c)

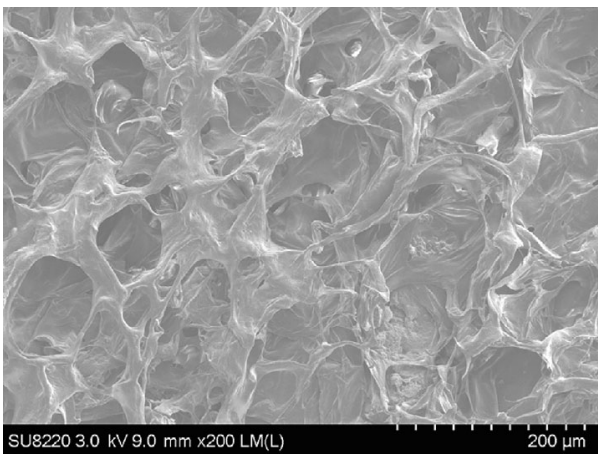

(e)

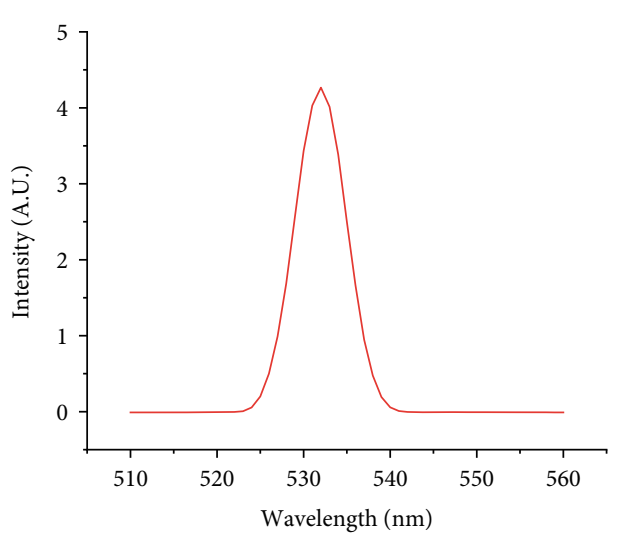

(g)

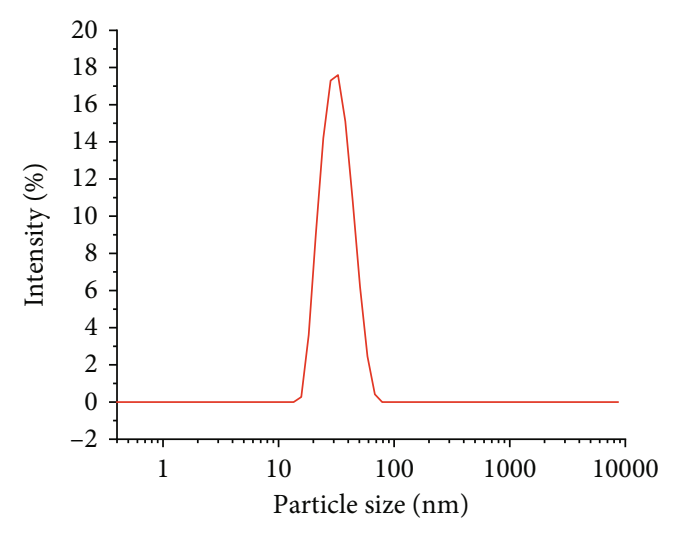

(b)

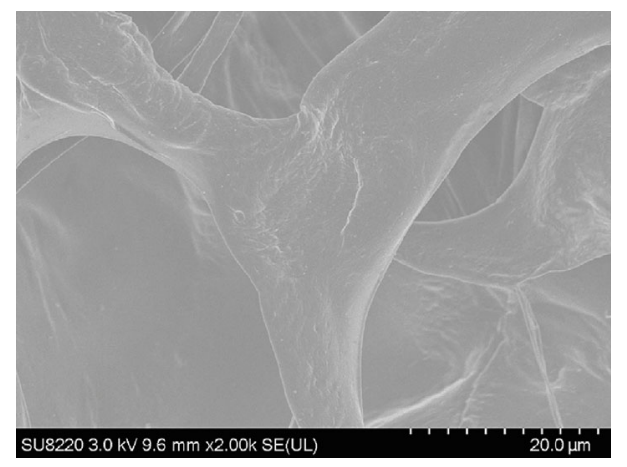

(d)

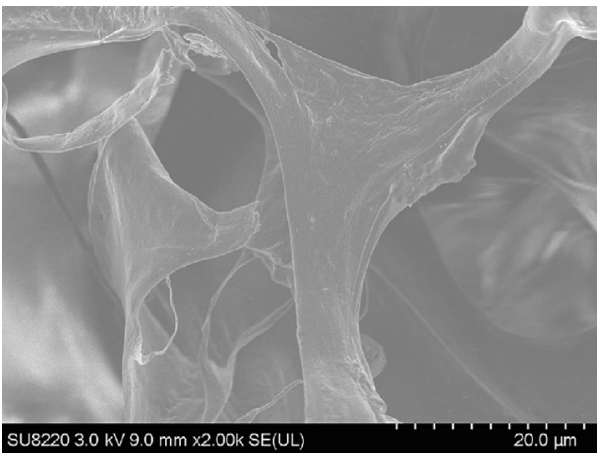

(f)

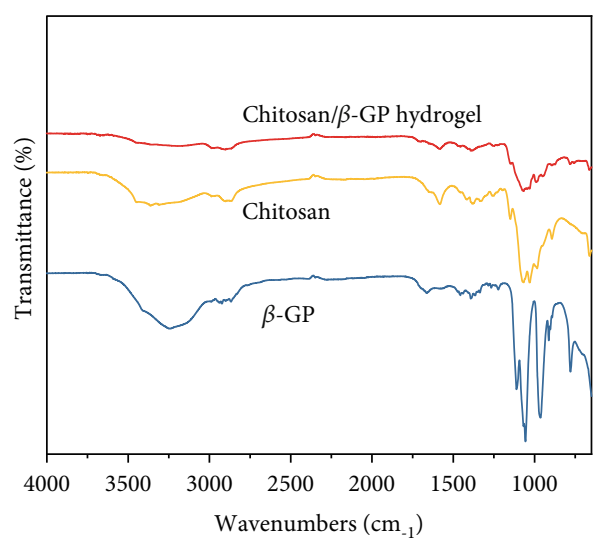

(h)

FIGURE 1: Characterization of UCNPs and UCNP/Chitosan/ $\beta$-GP hydrogel. (a). TEM images of UCNPs. (b). Particle size of UCNPs. SEM images of Chitosan/ $\beta$-GP hydrogel ( $c$ and d) and UCNP/Chitosan/ $\beta$-GP hydrogel (e and $\mathrm{f}$ ). (g). Photoluminescence intensity of UCNP/Chitosan/ $\beta$-GP hydrogel under $808 \mathrm{~nm}$ near-infrared light excitation. (h). FTIR analysis of Chitosan, $\beta$-GP, and Chitosan/ $\beta$-GP hydrogel. 


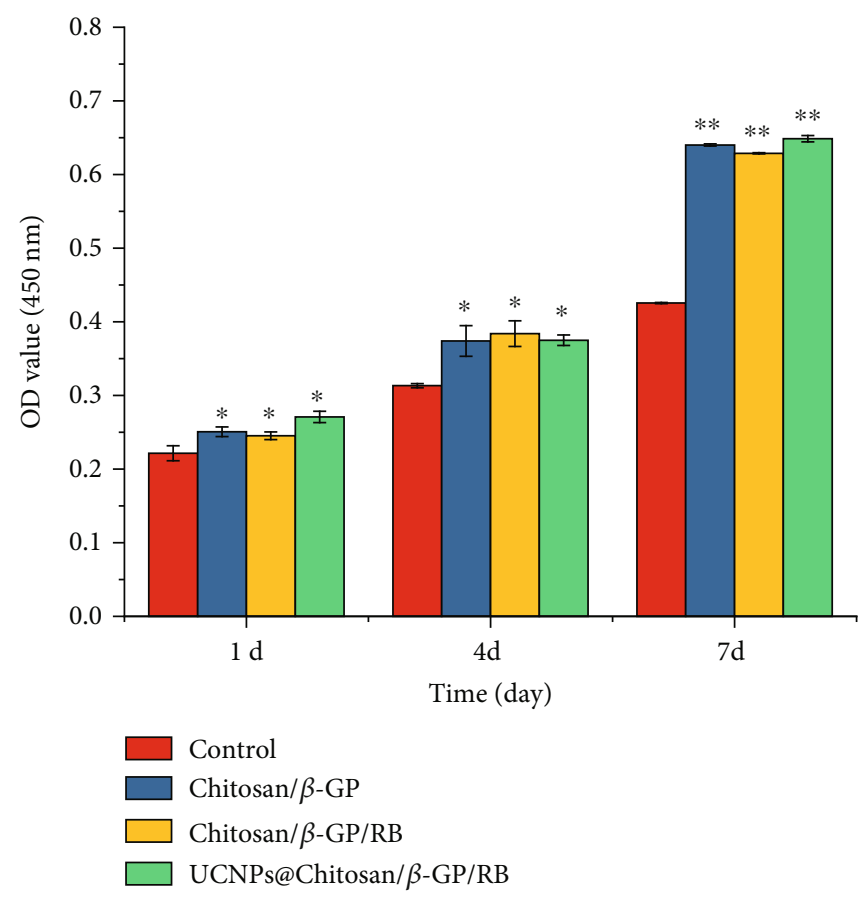

FIGURE 2: Cell viability analysis of human tenocytes incubated with different components in UCNP/RB/Chitosan/ $\beta$-GP hydrogel. ${ }^{*} p<0.05$ and ${ }^{* *} p<0.01$, compared with control.

transected surgically. In the SR group, the transected sites were repaired with standard Kessler suture. In the RB $+532 \mathrm{~nm}$ group, the transected site was treated with RB solution containing ROS Brite 700 (16004; AAT Bioquest, Sunnyvale, CA) and irradiated with $532 \mathrm{~nm}$ green light for $2 \mathrm{~min}$ for once. Then, the skin was sutured. In the UCNPs@hydrogel+808 nm group, the skin was firstly sutured after transection. Subsequently, UCNPs@RB/Chitosan/ $\beta$-GP hydrogel containing ROS Brite ${ }^{\mathrm{TM}} 700$ was injected into the transected site and irradiation with $808 \mathrm{~nm}$ near-infrared light was applied at 1, 4, and 7 days post operation. The rats without transection and irradiation were used as normal control.

2.6. Tunel Assay. The tissues $(1.5 \mathrm{~cm} \times 1.5 \mathrm{~cm} \times 0.3 \mathrm{~cm})$ were obtained from the ruptured Achilles tendon site and fixed in $10 \%$ formalin for $48 \mathrm{~h}$. Then, the tissues were embedded in paraffin and cut into $4 \sim 7 \mu \mathrm{m}$ slices. The cell death in different groups was detected using the In Situ Cell Death Detection Kit (11684817910, Roche, Mannheim, Germany) per the manufacturer's instruction.

2.7. Histological Assessment. The aforementioned $4 \sim 7 \mu \mathrm{m}$ slices were used for H\&E staining and Masson's trichrome staining. $\mathrm{H} \& \mathrm{E}$ staining was applied to assess the structure and arrangement of the fiber and cell density. Masson's trichrome staining was performed to evaluate collagen deposition at the transected site of the Achilles tendon.

2.8. Bioluminescence Imaging of ROS. All rats in the RB $+532 \mathrm{~nm}$ group and UCNPs@RB-hydrogel+808 nm group were probed with ROS Brite ${ }^{\mathrm{TM}} 700$. The bioluminescence imaging of ROS was performed on an in vivo imaging instru- ment (IVIS Lumina III; PerkinElmer, Waltham, MA, USA). After LED irradiation (680 nm wavelength), the $706 \mathrm{~nm}$ wavelength signals were acquired.

2.9. Statistical Analysis. The data was represented as mean \pm standard deviation. Statistical analysis was performed using SPSS 22.0. Statistical difference among groups was analyzed using one-way analysis of variance followed by Tukey's test. $P$ value $<0.05$ was considered as significant difference.

\section{Results}

3.1. Characterization of UCNPs and UCNP/Chitosan Hydrogel. As shown in Figures 1(a) and 1(b), the prepared UCNPs of $\mathrm{NaYF}_{4}: \mathrm{Yb} / \mathrm{Er}(\mathrm{Y}: \mathrm{Yb}: \mathrm{Er}=78: 20: 2)$ had a uniform shape with a particle size of $29.7 \pm 2.6 \mathrm{~nm}$. The addition of UCNPs did not affect the morphological structure of Chitosan/ $\beta$-GP hydrogel (Figures $1(\mathrm{c})$ and $1(\mathrm{~d})$ ). By adjusting the content of different components in the UCNPs, the UCNPs could convert the near-infrared light $(808 \mathrm{~nm})$ into green light $(532 \mathrm{~nm})$ (Figure $1(\mathrm{e}))$. Additionally, the FTIR spectra of Chitosan, $\beta-\mathrm{GP}$, and Chitosan/ $\beta-\mathrm{GP}$ are shown in Figure 1(f).

The cytotoxicity of Chitosan/ $\beta-\mathrm{GP}, \mathrm{RB} / \mathrm{Chitosan} / \beta-\mathrm{GP}$, and UCNPs@RB/Chitosan/ $\beta$-GP hydrogels in the tenocytes was assessed with the CCK- 8 assay. After 1, 4, and 7 days of incubation with Chitosan/ $\beta-\mathrm{GP}, \mathrm{RB} / \mathrm{Chitosan} / \beta-\mathrm{GP}$ and UCNPs@RB/Chitosan/ $\beta$-GP hydrogels, the cell viability of the tenocytes was even significantly higher than that in the control group (Figure 2). 

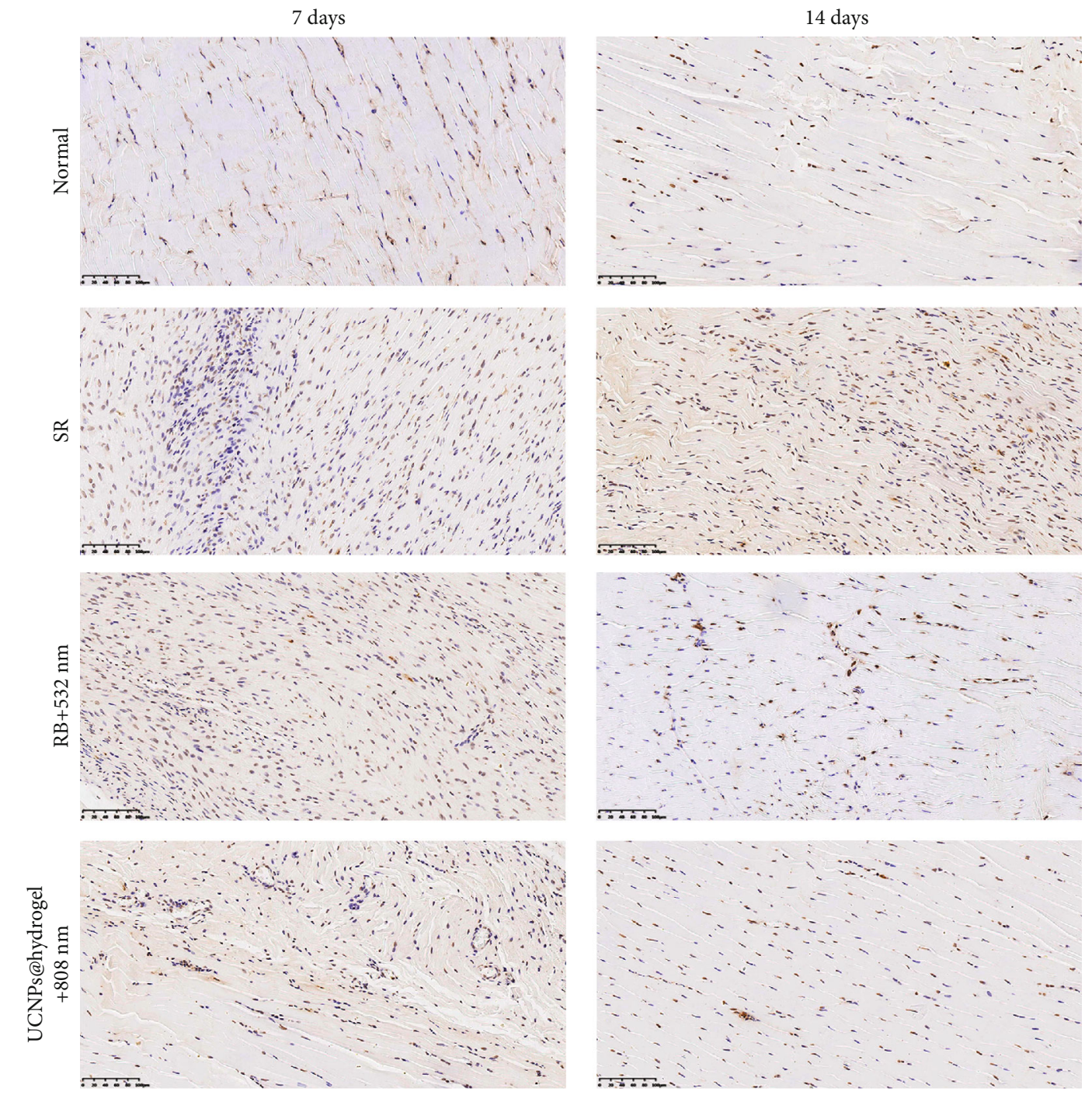

(a)

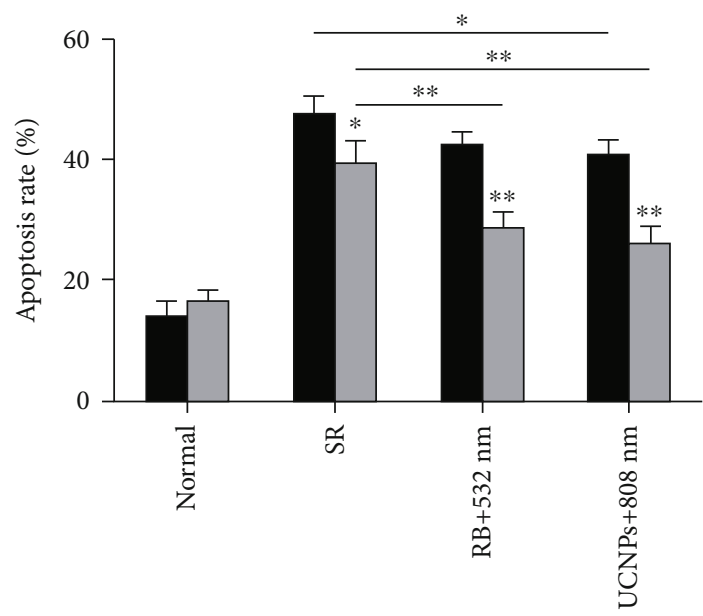

7 days
$\square$ days

(b)

FIgURE 3: The cell apoptosis for the Achilles tendon site in different groups at 7 days and 14 days post operation. (a) Representative images of cell apoptosis by Tunel assay. (b) The apoptosis rate analysis. ${ }^{*} p<0.05$ and ${ }^{* *} p<0.01$. 
7 days
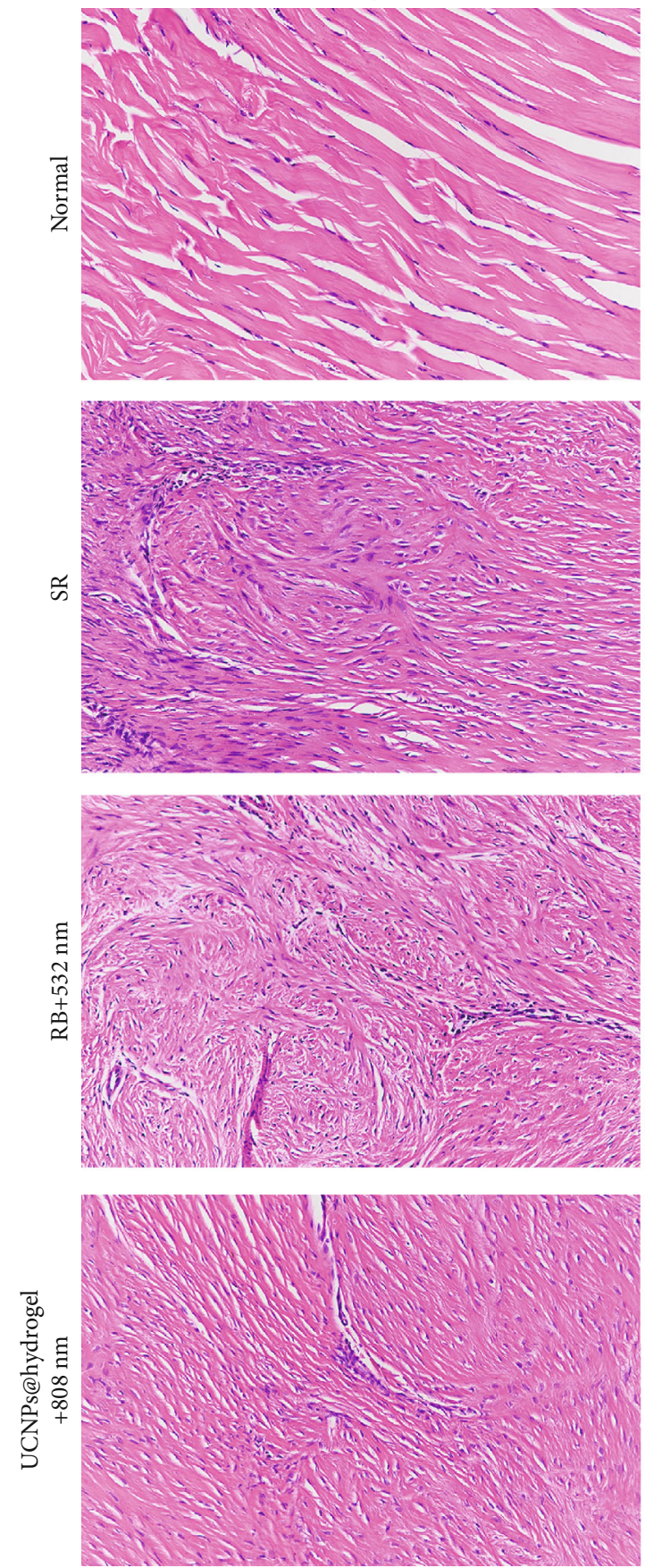

14 days
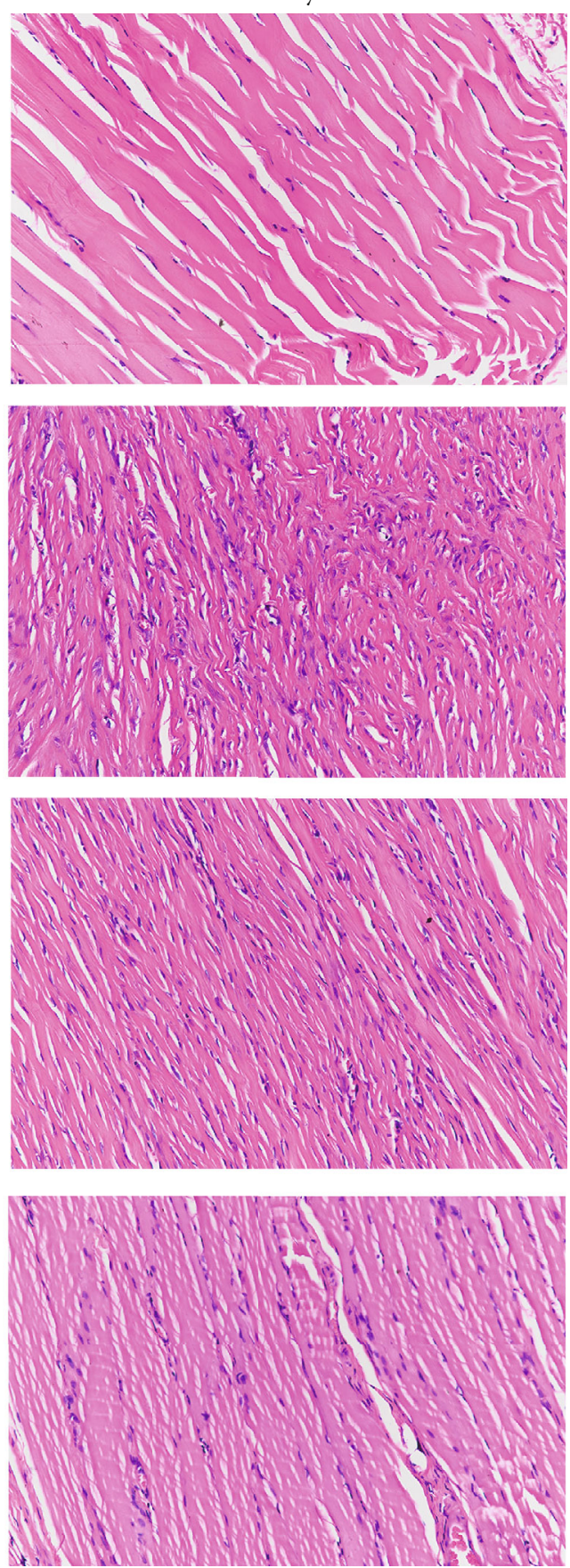

FIGURE 4: Representative images of hematoxylin and eosin staining for the Achilles tendon site in different groups at 7 days and 14 days post operation.

3.2. Observation of Cell Apoptosis. In the normal Achilles tendon, there are a small number of cells (Figure 3(a)). The number of live cells obviously increased in the three experimental groups (the SR, RB+532 nm, and UCNPs@hydrogel+808 nm groups) at 7 days post operation, while the cells were visibly reduced at 14 days post operation, especially in the $\mathrm{RB}$ $+532 \mathrm{~nm}$ group and the UCNPs@RB-hydrogel+808 nm group. Compared with the normal group, the three experimental groups had a significantly increased cell apoptosis rate at 7 days and 14 days post operation (Figure 3(b)). Compared with 7 days post operation, the cell apoptosis rate in all the three experimental groups decreased significantly at 14 days post operation. In the early stage of tendon repair, the cell apoptosis rate obviously increased, while, subsequently, the cell apoptosis rate obviously decreased. Notably, the apoptosis rate in the RB+532 nm and UCNPs@hydrogel+808 nm groups are much lower than that in the SR group at 14 days post operation, suggesting better effects on tendon repair. 

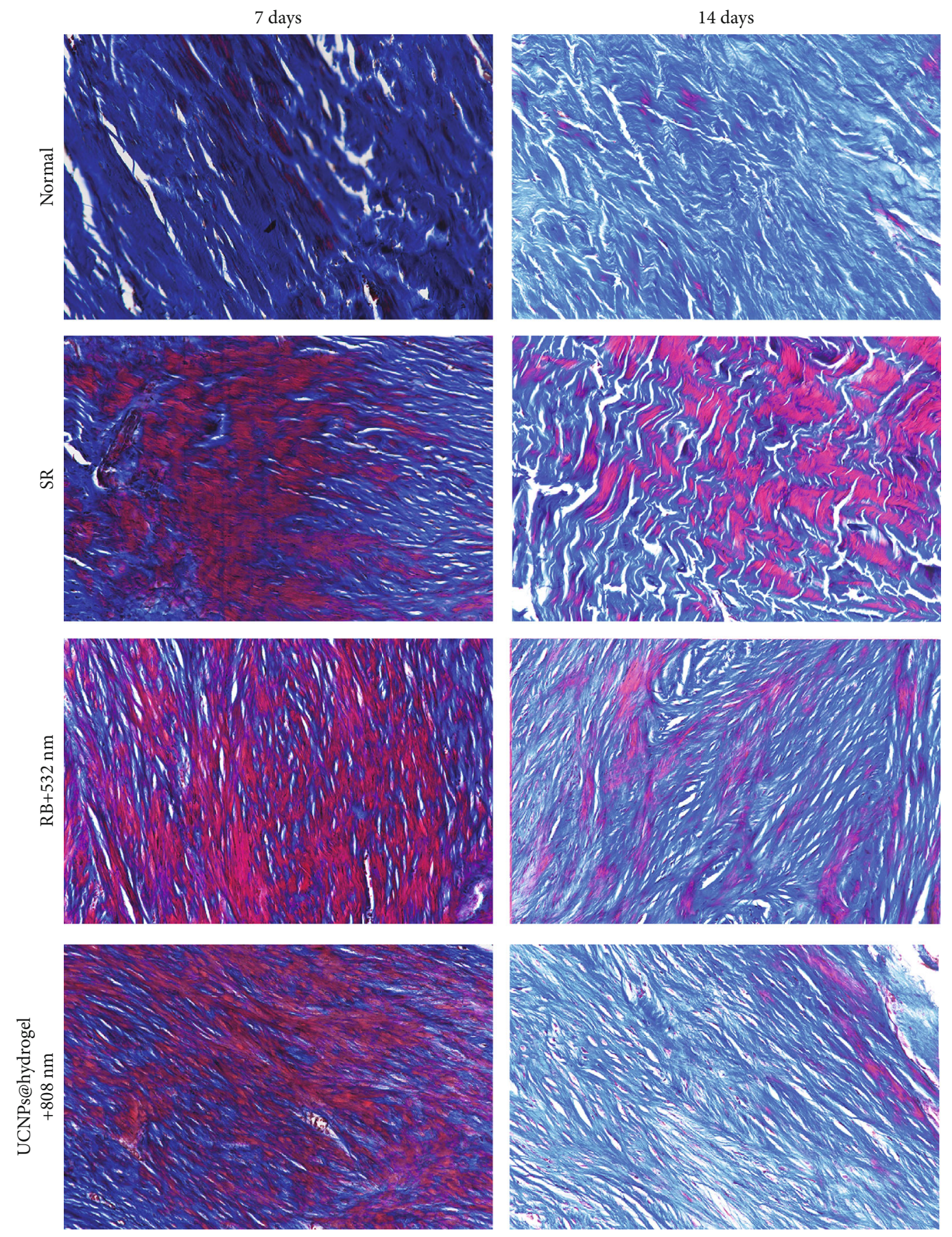

FIGURE 5: Representative images of Masson's trichrome staining for the Achilles tendon site in different groups at 7 days and 14 days post operation.

3.3. Histological Analysis of Achilles Tendon Healing. To evaluate the effect of different treatments on Achilles tendon repair, histological analysis was observed using H\&E staining and Masson's trichrome staining. H\&E staining showed that the normal Achilles tendon presented an integrated and continuous phenotype (Figure 4). At 7 days post operation, the Achilles tendon in the SR, RB+532 nm, and UCNPs@RBhydrogel $+808 \mathrm{~nm}$ groups appeared disordered. At 14 days post operation, the repaired Achilles tendon appeared much more integrated and continuous in the $\mathrm{RB}+532 \mathrm{~nm}$ and
UCNPs@RB-hydrogel+808 nm groups than in the SR group, especially in the UCNPs@RB-hydrogel+808 nm group.

Masson's trichrome staining showed that there were dense collagen fibers in the normal Achilles tendon, while the collagen fibers were much fewer in the SR, RB+532 nm, and UCNPs@RB-hydrogel+808nm groups at 7 days post operation (Figure 5). At 14 days post operation, the content of collagen fibers in the RB+532 nm and UCNPs@RB-hydrogel+808 $\mathrm{nm}$ groups was obviously higher than that in the SR group and was quite similar to that of the normal group. 

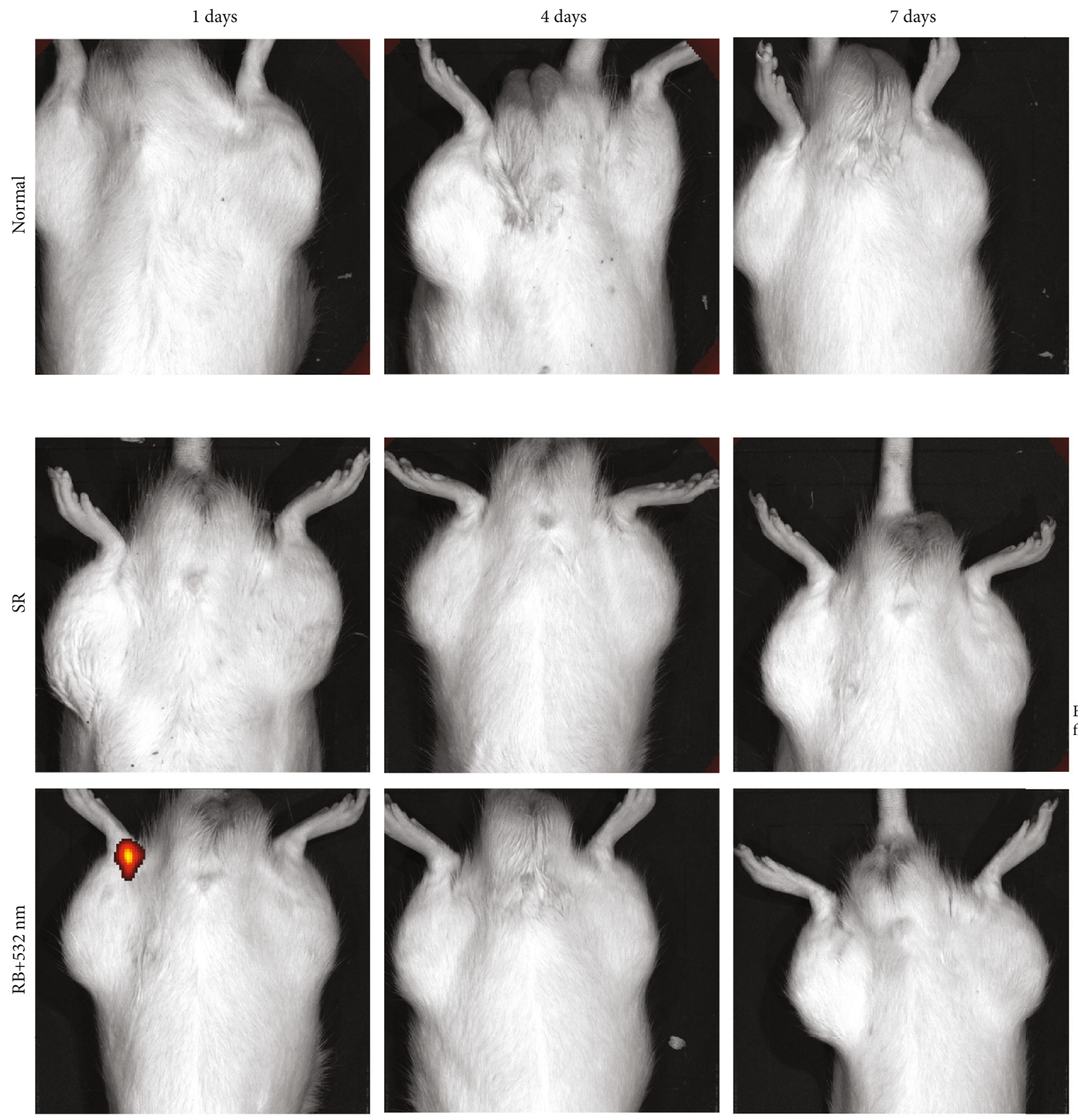

fluorescence
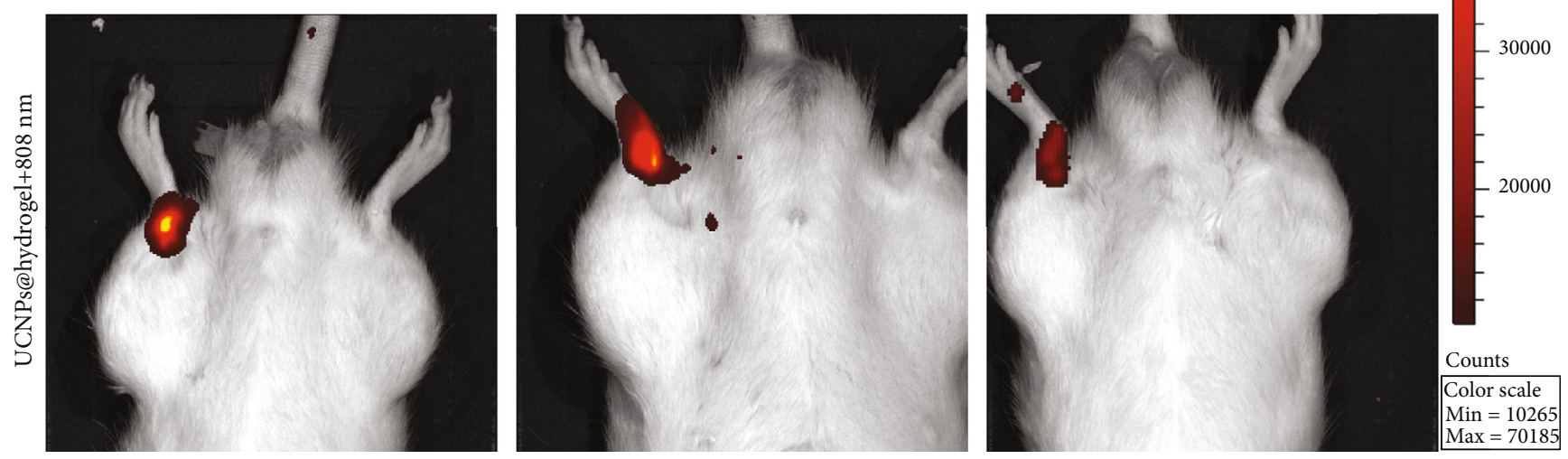

FIGURE 6: Bioluminescence imaging of ROS at the ruptured Achilles tendon site in different groups at 1, 4, and 7 days post operation. 
Moreover, compared with the $\mathrm{RB}+532 \mathrm{~nm}$ group, the collagen fibers were a little denser in UCNPs@RB-hydrogel +808 nm group.

3.4. Bioluminescence Imaging of ROS. As shown in Figure 6, there was no bioluminescence in the normal and SR groups. Bioluminescence was observed at 1 day post operation in the $\mathrm{RB}+532 \mathrm{~nm}$ group and at 1,4 , and 7 days post operation in the UCNPs@RB-hydrogel+808nm group. Moreover, the bioluminescence intensity in the UCNPs@RB-hydrogel $+808 \mathrm{~nm}$ group decreased markedly over time.

\section{Discussion}

Photochemical tissue bonding (PTB), based on photosensitizer rose bengal (RB) and green light, is an emerging suture-less biomedical application technology for tissue repair. PTB has been reported to be applied in numerous tissues, such as skin [6, 15-17], intestine [18], stomach [19], cornea [20, 21], nerves [22, 23], and blood vessels [24, 25]. For tendon repair, PTB has been reported to promote tendon repair at early stages in a full thickness Achilles tendon transection rat model [8]. Our previous study showed that the combination of PTB and electrospun silk provided benefits for tendon healing in an Achilles tendon transection rabbit model [9]. PTB also bonded human amniotic membrane with the surface of the flexor digitorum profundus tendon and thus promoted hand tendon repair better than surgical suture [10]. However, limited to the poor penetration of green light, while applying PTB in closed tendon rupture, the ruptured Achilles tendon has to be exposed by skin incision, which will cause secondary damage. This study explores the effects of noninvasive photochemical sealing on Achilles tendon rupture by a combination of PTB and UCNPs.

According to a previous study, compared with nontreated control, PTB treatment, which applied RB and green light, is beneficial for the repair of a transected Achilles tendon in rat [8]. In this study, evidenced by the results of Tunel assay, $H \& E$ staining, and Masson's trichrome staining, we demonstrated that in comparison with the traditional suture repair, the noninvasive photochemical sealing using modified PTB, which applied UCNPs@RB/Chitosan/ $\beta$-GP hydrogel and near-infrared light instead of green light, had better effects on Achilles tendon repair, while in comparison with the traditional PTB treatment, this noninvasive photochemical sealing had similar, even better effects on the repair of a transected Achilles tendon in the rat model.

Here, we also found that bioluminescence, which suggested the generation of ROS, was observed at 1 day post operation in the RB+532 nm group and at 1, 3, and 7 days post operation in the UCNPs@RB-hydrogel+808 nm group. Moreover, the bioluminescence intensity for ROS in the UCNPs@RB-hydrogel+808nm group decreased markedly over time. Our previous study showed that PTB treatment could significantly induce the generation of ROS and could promote cell proliferation and migration in tenocytes [26]. Thus, the effects of PTB or modified PTB with UCNPs might be attributed to the generation of ROS. Moreover, suitably more frequent generation of ROS in the UCNPs@RB-hydro- gel+808 nm group led to even better effects than that for only once in the $\mathrm{RB}+532 \mathrm{~nm}$ group.

In conclusion, injectable hydrogels containing $\mathrm{RB}$ and UCNPs (UCNPs@RB/Chitosan/ $\beta$-GP hydrogel), which could convert near-infrared light to green light, were prepared and were not toxic to tenocytes. Based on the peculiarity of UCNPs, PTB technology was modified and exerted its role on the site of a transected Achilles tendon without incising skin. Therefore, the combination of PTB and UCNPs@RB/Chitosan/ $\beta$-GP hydrogel could be used as a noninvasive photochemical sealing for Achilles tendon rupture.

\section{Data Availability}

The data used to support the findings of this study are included within the article.

\section{Conflicts of Interest}

The authors report no conflict of interest.

\section{Authors' Contributions}

Yiming Zhu and Aiguo Xie contributed equally to this work and they are co-first authors.

\section{Acknowledgments}

This work was supported by the National Science Foundation of China under Grant No. 81401585.

\section{References}

[1] A. Ganestam, T. Kallemose, A. Troelsen, and K. W. Barfod, "Increasing incidence of acute Achilles tendon rupture and a noticeable decline in surgical treatment from 1994 to 2013. A nationwide registry study of 33,160 patients," Knee surgery, sports traumatology, arthroscopy, vol. 24, no. 12, pp. 37303737, 2016.

[2] K. RJK and R. L. Carey Smith, "Surgical interventions for treating acute Achilles tendon ruptures," The Cochrane database of systematic reviews, no. article CD003674, 2010.

[3] N. Jiang, B. Wang, A. Chen, F. Dong, and B. Yu, "Operative versus nonoperative treatment for acute Achilles tendon rupture: a meta-analysis based on current evidence," International Orthopaedics, vol. 36, no. 4, pp. 765-773, 2012.

[4] R. Wilkins and L. J. Bisson, "Operative versus nonoperative management of acute Achilles tendon ruptures: a quantitative systematic review of randomized controlled trials," The American journal of sports medicine, vol. 40, no. 9, pp. 2154-2160, 2012.

[5] Y. Ochen, R. B. Beks, M. van Heijl et al., "Operative treatment versus nonoperative treatment of Achilles tendon ruptures: systematic review and meta-analysis," BMJ, vol. 364, p. k5120, 2019.

[6] S. Tsao, M. Yao, H. Tsao et al., "Light-activated tissue bonding for excisional wound closure: a split-lesion clinical trial," The British journal of dermatology, vol. 166, no. 3, pp. 555-563, 2012.

[7] N. Vanerio, M. Stijnen, B. de Mol, and L. M. Kock, "Biomedical Applications of Photo- and Sono-Activated rose bengal: A 
Review," Photobiomodulation, photomedicine, and laser surgery, vol. 37, no. 7, pp. 383-394, 2019.

[8] B. P. Chan, C. Amann, A. N. Yaroslavsky et al., "Photochemical repair of Achilles tendon rupture in a rat model," The Journal of surgical research, vol. 124, no. 2, pp. 274-279, 2005.

[9] T. Ni, P. Senthil-Kumar, K. Dubbin et al., "A photoactivated nanofiber graft material for augmented Achilles tendon repair," Lasers in surgery and medicine, vol. 44, no. 8, pp. 645-652, 2012.

[10] B. Ding, X. Wang, and M. Yao, "Photochemical Tissue Bonding Technique for Improving Healing of Hand Tendon Injury," Surgical innovation, vol. 26, no. 2, pp. 153-161, 2018.

[11] J. Zhang, C. Mi, H. Wu, H. Huang, C. Mao, and S. Xu, "Synthesis of NaYF4: $\mathrm{Yb} / \mathrm{Er} / \mathrm{Gd}$ up-conversion luminescent nanoparticles and luminescence resonance energy transfer-based protein detection," Analytical Biochemistry, vol. 421, no. 2, pp. 673679, 2012.

[12] S. Han, B. W. Hwang, E. Y. Jeon et al., "Upconversion nanoparticles/hyaluronate-rose bengal conjugate complex for noninvasive photochemical tissue bonding," ACS Nano, vol. 11, no. 10, pp. 9979-9988, 2017.

[13] A. Chenite, C. Chaput, D. Wang et al., "Novel injectable neutral solutions of chitosan form biodegradable gels in situ," Biomaterials, vol. 21, no. 21, pp. 2155-2161, 2000.

[14] G. Molinaro, J. C. Leroux, J. Damas, and A. Adam, "Biocompatibility of thermosensitive chitosan-based hydrogels: an in vivo experimental approach to injectable biomaterials," Biomaterials, vol. 23, no. 13, pp. 2717-2722, 2002.

[15] B. P. Chan, I. E. Kochevar, and R. W. Redmond, "Enhancement of porcine skin graft adherence using a light-activated process," The Journal of surgical research, vol. 108, no. 1, pp. 77-84, 2002.

[16] Y. Kamegaya, W. A. Farinelli, A. V. Vila Echague et al., "Evaluation of photochemical tissue bonding for closure of skin incisions and excisions," Lasers in surgery and medicine, vol. 37, no. 4, pp. 264-270, 2005.

[17] P. Yang, M. Yao, S. L. DeMartelaere, R. W. Redmond, and I. E. Kochevar, "Light-activated sutureless closure of wounds in thin skin," Lasers in surgery and medicine, vol. 44, no. 2, pp. 163-167, 2012.

[18] A. Lauto, D. Mawad, M. Barton, A. Gupta, S. C. Piller, and J. Hook, "Photochemical tissue bonding with chitosan adhesive films," Biomedical engineering online, vol. 9, no. 1, p. 47, 2010.

[19] M. Ark, P. Boughton, A. Lauto et al., "Characterisation of a novel light activated adhesive scaffold: potential for device attachment," Journal of the mechanical behavior of biomedical materials, vol. 62, pp. 433-445, 2016.

[20] E. E. Verter, T. E. Gisel, P. Yang, A. J. Johnson, R. W. Redmond, and I. E. Kochevar, "Light-initiated bonding of amniotic membrane to cornea," Investigative ophthalmology \& visual science, vol. 52, no. 13, pp. 9470-9477, 2011.

[21] H. Zhu, C. Alt, R. H. Webb, S. Melki, and I. E. Kochevar, "Corneal crosslinking with rose bengal and green light: efficacy and safety evaluation," Cornea, vol. 35, no. 9, pp. 12341241, 2016.

[22] T. S. Johnson, A. C. O’Neill, P. M. Motarjem et al., "Photochemical tissue bonding: a promising technique for peripheral nerve repair," The Journal of surgical research, vol. 143, no. 2, pp. 224-229, 2007.

[23] A. C. O’Neill, M. A. Randolph, K. E. Bujold, I. E. Kochevar, R. W. Redmond, and J. M. Winograd, "Preparation and integra- tion of human amnion nerve conduits using a light-activated technique," Plastic and reconstructive surgery, vol. 124, no. 2, pp. 428-437, 2009.

[24] A. C. O’Neill, J. M. Winograd, J. L. Zeballos et al., "Microvascular anastomosis using a photochemical tissue bonding technique," Lasers in surgery and medicine, vol. 39, no. 9, pp. 716-722, 2007.

[25] P. Senthil-Kumar, J. H. Ng-Glazier, M. A. Randolph et al., “An intraluminal stent facilitates light-activated vascular anastomosis," The journal of trauma and acute care surgery, vol. 83, 1 Suppl 1, pp. S43-S49, 2017.

[26] Z. Yao, X. Wang, W. Zhang, Y. Liu, and T. Ni, "Photochemical tissue bonding promotes the proliferation and migration of injured tenocytes through ROS/RhoA/NF- $\kappa$ B/Dynamin 2 signaling pathway," Journal of cellular physiology, vol. 233, no. 10, pp. 7047-7056, 2018. 